\title{
Argus II retinal prosthesis malrotation and repositioning with intraoperative optical coherence tomography in a posterior staphyloma
}

This article was published in the following Dove Press journal:

Clinical Ophthalmology

24 November 2015

Number of times this article has been viewed

\author{
Michael I Seider \\ Paul Hahn \\ Duke University Eye Center, \\ Durham, NC, USA
}

Correspondence: Paul Hahn

Duke University Eye Center, 235I Erwin

Road, Durham, NC 27705, USA

$\mathrm{Tel}+|9| 9684563$ |

Fax +19196816474

Email paul.s.hahn@duke.edu
Introduction: The Argus II retinal prosthesis may improve visual function in patients with severe vision loss from retinitis pigmentosa. Optimal centration of the electrode array over the macula is important to achieve optimal visual results. Argus tack malrotation is a novel entity that may be encountered during placement, especially in patients with posterior staphyloma.

Methods: Retrospective case review.

Results: During tacking of the electronics array a clockwise rotation occurred resulting in malposition. We hypothesize this was secondary to undue rotation or posterior pressure applied during tack insertion in conjunction with placement over a previously unrecognized posterior staphyloma. Intraoperative optical coherence tomography, because of the cross-sectional images provided, was helpful in visualizing the distance between the electronics array and the retina, which was difficult to assess using the surgical microscope alone. Repositioning was achieved by adjusting the tack without removal. The patient experienced an improvement in vision as a result of the surgery.

Conclusion: Malrotation may occur when tacking the Argus II prosthesis, and the presence of a posterior staphyloma may increase this risk. It is important to differentiate malrotation from tack misplacement - the former may be addressed with array unrotation or partial tack withdrawal and the latter may require tack removal and reinsertion. Also, intraoperative optical coherence tomography may be helpful in characterizing electronics array position during surgery.

Keywords: Argus, retinitis pigmentosa, retinal tack

\section{Introduction}

The Argus II Retinal Prosthesis System (Second Sight Medical Products Inc., Sylmar, CA, USA) may improve visual function in patients with end-stage retinitis pigmentosa. ${ }^{1}$ Visual outcomes likely depend on centration and apposition of the electronics array to the macula. ${ }^{2,3} \mathrm{~A}$ few cases of array malpositioning that were identified postoperatively have been reported. ${ }^{2}$ In some of these cases, a subsequent surgical procedure with tack withdrawal was performed to reposition the array and improve visual outcome.

A posterior staphyloma alters the normal contour of the posterior eye wall and may adversely influence proper centration and apposition of a semi-rigid array. ${ }^{1} \mathrm{We}$ present a case of Argus II implantation in a patient with a posterior staphyloma who experienced intraoperative tack malrotation and who achieved array centration and partial apposition via guidance during surgery by feedback from direct visualization and intraoperative optical coherence tomography (OCT).

\section{Results}

The Argus II epiretinal prosthesis was placed in the left eye of a 66-year-old man with bare light-perception vision in each eye from retinitis pigmentosa (Figure 1A). 
Preoperatively, spectral-domain OCT (Heidelberg Engineering, Heidelberg, Germany) demonstrated a posterior staphyloma (Figure 1B). During surgery, at the final moment of tack insertion, unintentional rotation of the array occurred (Figure $1 \mathrm{C}$ ), resulting in the array partially overlying the optic nerve instead of being centered in the macula. The reason for this malrotation is unclear. We hypothesize this occurred because of an unintentional manual clockwise rotation of the forceps during insertion. It is also possible that excessive posterior pressure during tack placement resulted in tack rotation from over-compression of the tack spring. The position of the electronics array is determined by the orientation of the electronics cable as it enters the sclera, and it is also possible that array rotation occurred because of uneven tension on this cable due to an oblique sclerotomy.

In addition to poor centration, intraoperative OCT (Bioptigen Inc., Morrisville, NC, USA) revealed the electrode array to be elevated by the edge of the macular staphyloma and poorly apposed to the retinal surface (Figure 1D), which was not appreciated with the surgical microscope alone. At the time of surgery, prior to reviewing the surgical video, poor centration of the electronics array was attributed to tack misplacement rather than array malrotation. Attempts were made initially to withdraw and replace the tack. The tack was unable to be withdrawn with moderate force. As the tack was released from the tack forceps, the array was noted to unrotate, leaving the array still anchored by the tack and optimally centered over the macula (Figure 1E). We hypothesize this occurred due to the partial release of compressive tack pressure. Intraoperative OCT was then performed again demonstrating the array to be free from the staphyloma edge (Figure 1F). Three months postoperatively, the implant remained well centered in the macula and free from the staphyloma edge (Figure 1G) (Video S1). The array remained elevated up to $500 \mu \mathrm{m}$ from the retinal surface postoperatively (Figure 1H). Despite the distance of the array from the retinal surface, the patient has done well with a significant subjective improvement in function following activation of the device. In addition, objective tests performed 1 year following surgery revealed improvements in visual function when the device was active. On square localization testing, the patient scored $7.5 \%$ (3/40) with the device turned off and 32.5\% (13/40) with the device turned on. On direction of motion testing the patient scored $6.3 \%(5 / 80)$ with the device turned off and $12.5 \%(10 / 80)$ with the device turned on. The patient did not have appreciable improvements in grated visual acuity with device activation.
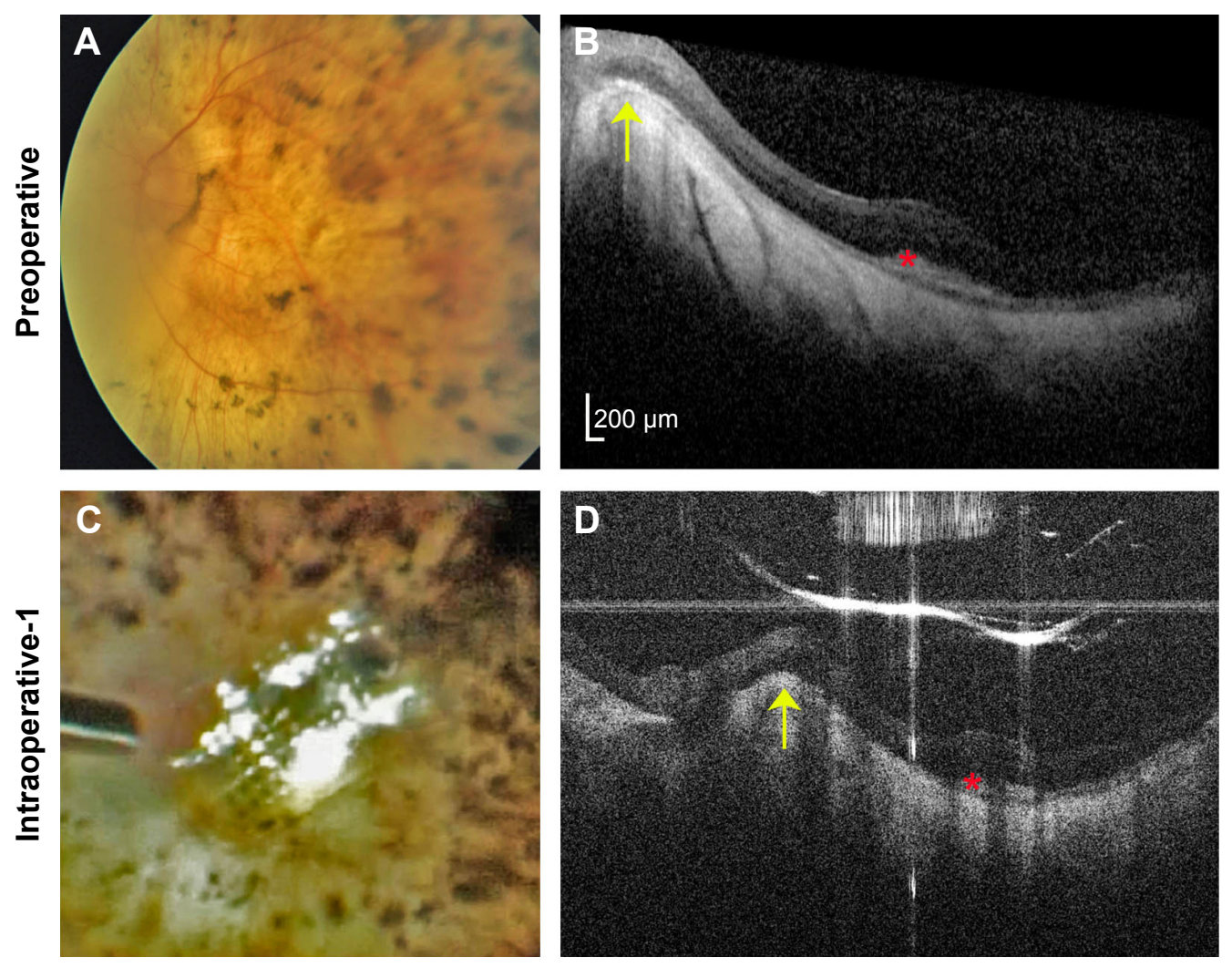

Figure I (Continued) 

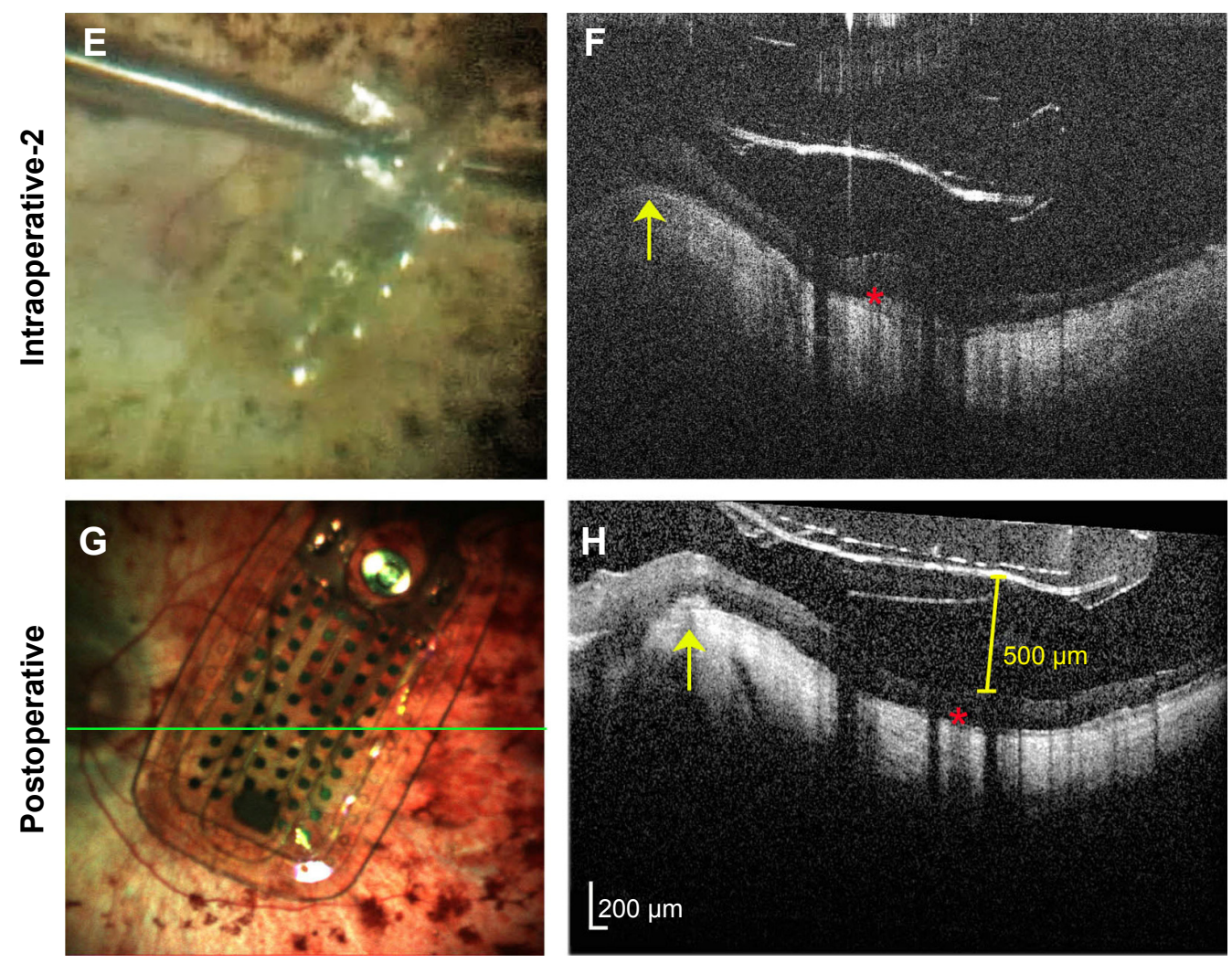

Figure I Argus II prosthesis repositioning.

Notes: (A) Preoperative color fundus photo of the left eye of a 66-year-old man with end-stage retinitis pigmentosa. (B) Preoperative spectral-domain optical coherence tomography (OCT) image showing sloping of the eye wall consistent with a posterior staphyloma. The elevated edge of the staphyloma (yellow arrow) and sub-retinal hyperreflective material (red asterisk) are also in subsequent OCT images (D, F, and $\mathbf{H}$ ) as landmarks showing similar scan locations. Note that all OCT images are shown in the standard format with elongated axial dimensions that exaggerate the distance between the prosthesis and the retinal surface. (C) Intrasurgical video-still image showing array malrotation over the optic nerve. (D) Intraoperative hand-held spectral-domain OCT image captured just after the moment in surgery imaged in (C), showing the array proppedup by the edge of the staphyloma. (E) Intrasurgical video-still image after array unrotation showing optimal positioning in the macula. (F) Intraoperative hand-held spectral-domain OCT captured just after the moment in surgery imaged in (E), showing the array free from the staphyloma edge. (G) Color scanning laser ophthalmoscopy image captured 3 months after surgery showing sustained optimal array positioning. The green line represents the OCT scan location shown in $(\mathbf{H})$. $(\mathbf{H})$ Spectral-domain OCT image 3 months after surgery confirming the array remains free from the staphyloma edge. The array does not fully contact the retina, and maximal distance between the array and retinal surface measures $500 \mu \mathrm{m}$ (yellow calipers).

\section{Conclusion}

The presence of a posterior staphyloma may affect optimal implantation of the Argus II prosthesis. ${ }^{1}$ We suggest that the surgical plan for placement in patients with a posterior staphyloma account for curvature in the eye wall to maximize proximity of the semi-rigid array to the retinal surface. Intraoperative OCT enables cross-sectional visualization during surgery and may be helpful in visualizing axial proximity of the implant to the retina, which is otherwise difficult to assess using the surgical microscope alone. In addition, intraoperative OCT may permit identification of anatomic variations and/or pathology (posterior staphyloma, epiretinal membranes, residual posterior vitreous, etc), which may also affect surgical decision-making.

Also, unintended rotation of an array during implantation can occur. It is important to differentiate malrotation from tack misplacement - the former may be addressed with array unrotation or partial tack withdrawal and the latter may require tack removal and reinsertion. In cases of staphylomas, complete apposition of the Argus array to the retinal surface may not be possible although improvements in visual function may still be obtained in such patients, as Rizzo et al have also shown. ${ }^{1}$ Argus II electronics array positioning can be optimized by direct visualization and intraoperative OCT guidance. Our patient has achieved meaningful visual and functional gains, suggesting that the presence of a staphyloma and reduced array-retina contact should not be a contraindication to Argus II implantation.

\section{Acknowledgment}

This manuscript was supported by the Heed Ophthalmic Foundation.

\section{Disclosure}

Paul Hahn is a paid consultant for Second Sight Medical Products, Inc. (Sylmar, CA, USA). The authors report no other disclosures or pertinent conflicts of interest in this work. 


\section{References}

1. Rizzo S, Belting C, Cinelli L, et al. The Argus II retinal prosthesis: 12-month outcomes from a single-study center. Am J Ophthalmol. 2014; 157(6):1282-1290.

2. de Juan E, Spencer R, Barale PO, da Cruz L, Neysmith J. Extraction of retinal tacks from subjects implanted with an epiretinal visual prosthesis. Graefes Arch Clin Exp Ophthalmol. 2013;251(10):2471-2476.
3. Ahuja AK, Yeoh J, Dorn JD, et al. Factors affecting perceptual threshold in Argus II retinal prosthesis subjects. Transl Vis Sci Technol. 2013; 2(4):1.

\section{Publish your work in this journal}

Clinical Ophthalmology is an international, peer-reviewed journal covering all subspecialties within ophthalmology. Key topics include: Optometry; Visual science; Pharmacology and drug therapy in eye diseases; Basic Sciences; Primary and Secondary eye care; Patient Safety and Quality of Care Improvements. This journal is indexed on

\section{Dovepress}

PubMed Central and CAS, and is the official journal of The Society of Clinical Ophthalmology (SCO). The manuscript management system is completely online and includes a very quick and fair peer-review system, which is all easy to use. Visit http://www.dovepress.com/ testimonials.php to read real quotes from published authors. 